\title{
Intraprocedural Flat Panel Detector Rotational Angiography and an Image Fusion Technique for Delivery of a Microcatheter into the Targeted Shunt Pouch of a Dural Arteriovenous Fistula
}

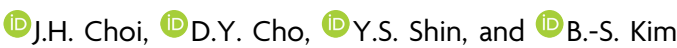

\begin{abstract}
SUMMARY: The accurate and safe delivery of a microcatheter to a targeted shunt pouch is essential for successful transvenous embolization of intracranial dural arteriovenous fistulas. However, complex anatomy and variations in head and neck veins and occluded sinuses can hinder intraprocedural microcatheter delivery. In this study, we introduce an intraprocedural flat panel detector rotational angiography and image fusion technique to aid precise navigation inside the veins and proper placement of the microcatheter in the targeted shunt pouch.
\end{abstract}

ABBREVIATIONS: $\mathrm{ACC}=$ anterior condylar confluence; $\mathrm{DAVF}=$ dural arteriovenous fistula; FPCTRA $=$ flat panel CT rotational angiography; IF $=$ image fusion; TVE = transvenous embolization

$F$ or the successful treatment of intracranial dural arteriovenous fistulas (DAVFs), precise evaluation of the angioarchitecture using pretherapeutic DSA is important. However, identification of detailed angioarchitectures of some DAVFs using conventional 2D-DSA or 3D rotational angiography images is difficult because of overlapping vessels. ${ }^{1,2}$ With the evolution of angiography equipment and $3 \mathrm{D}$ postprocessing technology, performing a flat panel CT rotational angiography and image fusion (FPCTRA and IF) technique to obtain a fusion image using 2 different rotational angiographic images is feasible. This technique enables physicians to gain better pretherapeutic knowledge for establishing an appropriate treatment plan. ${ }^{1-3}$ However, to our knowledge, no research has focused mainly on the value of the intraprocedural FPCTRA and IF technique. Especially, for transvenous embolization (TVE), the most important factor for successful occlusion is the accurate and safe delivery of a microcatheter to the shunt pouch. Complex anatomy and variations in head and neck veins, ${ }^{4}$ especially those located in the skull base, ${ }^{5}$ and sinus occlusion ${ }^{6,7}$

Received April 3, 2020; accepted after revision June 3.

From the Departments of Neurosurgery (J.H.C., Y.S.S.) and Radiology (B.-S.K.), Seoul St. Mary's Hospital, The Catholic University of Korea College of Medicine, Seoul, Republic of Korea; Department of Neurosurgery (D.Y.C.), Ewha Womans University Seoul Hospital, College of Medicine, Ewha Womans University, Seoul, Republic of Korea.

Please address correspondence to Bum-Soo Kim, MD, PhD, Department of Radiology, Seoul St. Mary's Hospital, The Catholic University of Korea, 222 Banpodaero, Seochogu, Seoul, 06591, Republic of Korea; e-mail: bumrad@catholic.ac.kr

Indicates article with supplemental on-line appendix and table.

Indicates article with supplemental on-line photos.

http://dx.doi.org/10.3174/ajnr.A6724

can hinder intraprocedural microcatheter delivery. In this study, we introduce an intraprocedural FPCTRA and IF technique to aid the navigation of a microcatheter inside veins and confirm the placement of the microcatheter in the targeted pouch.

\section{Technique}

For treating all DAVFs and image processing, we used Artis Zee biplane angiography equipment (Siemens). All procedures were conducted with the patient under general anesthesia. Systemic heparin (2000- to 3000-IU bolus) was routinely administrated after placement of a femoral sheath. Antiplatelet agents were not routinely administrated. We placed a $5 \mathrm{~F}$ angiocatheter in the main feeding artery and a 7F guiding catheter in the internal jugular vein or the retromandibular vein according to the access route. Thereafter, we performed $3 \mathrm{D}$ dual rotation angiography with contrast injection to obtain subtraction (vessel only), mask (bone only), and native fill (vessel with bone) images as a baseline. We named this series DSA24, which irradiated at $0.24 \mu \mathrm{Gy} /$ frame and took 30 frame/s for 5 seconds. The rotational angle was $200^{\circ}$ with $1.5^{\circ}$ increments, resulting in 133 projections and a rotation velocity of $40 \%$ s. For contrast, we infused iodixanol (Visipaque 270; GE Healthcare) via the external carotid artery with an injector at a rate of $2-3 \mathrm{~mL} / \mathrm{s}$ for 5 seconds with a 1- to 2second acquisition delay according to vessel diameter and shunt flow. Acquired data were sent to the workstation (syngo 3D workplace; Siemens), and 3D reconstruction was performed with the following parameters: kernel type, Hounsfield unit; image characteristics, normal; reconstruction mode, dual volume; section matrix, $512 \times 512$; and voxel size, $0.47 \mathrm{~mm}$. A size 18 


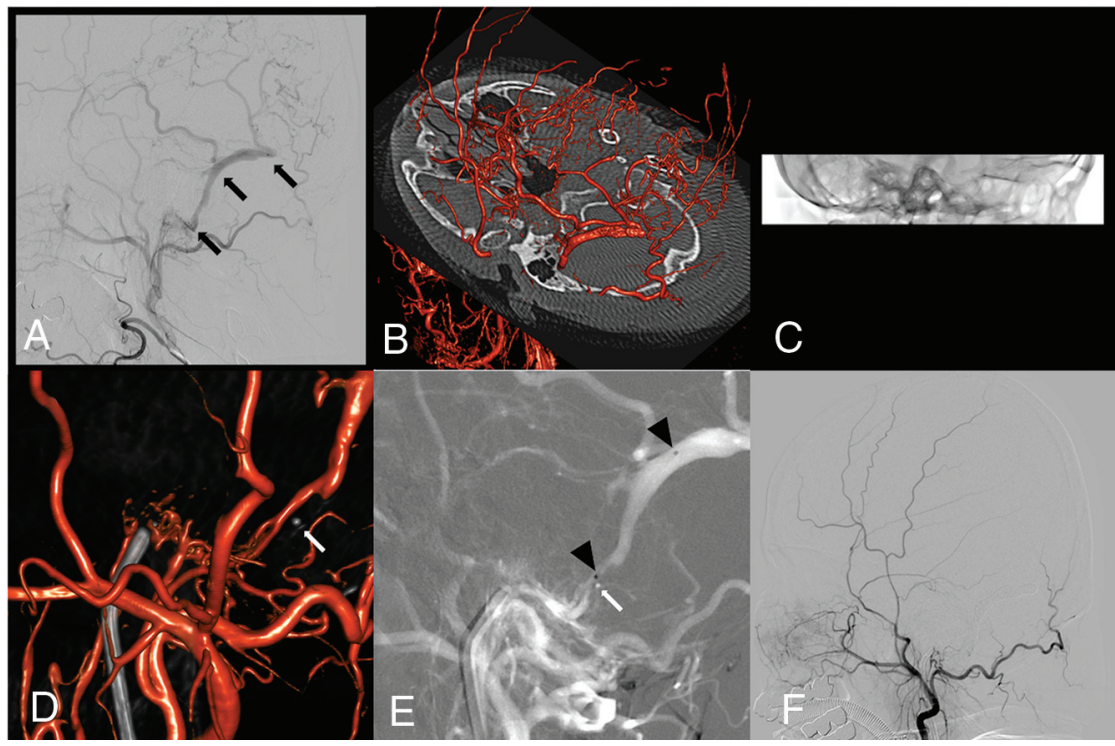

FIGURE. Case 1. A 61-year-old female patient presenting with intracranial hemorrhage. A, Initial right external carotid angiography shows a transverse sigmoid dural AVF with an isolated sinus (black arrow) and pial reflux. $B$, Intraprocedural $0.24-\mu \mathrm{G} /$ frame FPCTRA (DSA24) is performed to obtain subtraction and mask images as a control. $C$, During the microcatheter navigation, we perform low-dose FPCTRA with a reduced FOV (DRcare). D, We found that the microcatheter was placed in the wrong direction (white arrow) by fusion of the initial DSA24 and follow-up DRcare images. E, After we pulled back the microcatheter (white arrow), we directed it the right way and placed the microcatheter in the target shunt (black arrowhead). F, Successful coil embolization is performed.

microcatheter (Excelsior 1018, Stryker) and a 0.014-inch microwire (Transend; Stryker) were usually used for navigation. During navigation, if needed, we performed 3D single-rotation angiography with a reduced FOV without contrast injection while keeping the microwire and microcatheter inside the vein and fused with the initial subtraction image to identify the microcatheter going in the right direction. This series was named DRcare, which was performed with a single rotation for 5 seconds and irradiated $0.10 \mu \mathrm{Gy} /$ frame. In the workstation, we performed $3 \mathrm{D}$ reconstruction: kernel type, EE; image characteristics, normal; reconstruction mode, native. Two different image datasets (DSA24 and DRcare) were fused by a mapping application syngo iDentify (Syngo 3D workplace, Siemens AG, Erlangern, Germany), which superimposes the spatial coordinate system of the initial dataset onto that of the second dataset. We used landmark-based automatic registration to avoid misalignment. ${ }^{8}$ When the microcatheter accessed the shunt pouch, we also checked DRcare and fused images to confirm the exact placement of the microcatheter in the target. Thereafter, we inserted the coil until the shunt disappeared.

\section{Clinical Results}

Details of the study population and outcome assessment are presented in the On-line Appendix. We used the FPCTRA and IF technique in 18 of 58 DAVFs treated with TVE between January 2009 and March 2020. Among them, 11 had ipsilateral sinus occlusion ( 8 in the cavernous sinus, 2 in the anterior condylar confluence [ACC], and 1 in the transverse-sigmoid sinus). We approached the shunt pouch through the occluded sinus by blinded navigation $(n=6)$ or through alternative venous routes (the facial or superficial temporal vein, $n=5)$. Seven DAVFs were located in the ACCs. The complete or near-complete occlusion rate and symptom-free or improved rate were $94.4 \%$ and $100 \%$, respectively. There was no catheter-related vessel injury with or without using this technique. We could access the shunt via the transfemoral route in all cases treated using this technique (On-line Table). In contrast, we failed to reach the shunt pouch via the transfemoral approach in 5 cases without this technique. Therefore, surgical cannulation of the target sinus or the direct ophthalmic vein approach was additionally performed to reach the shunt. Illustrative cases are presented in the Figure and On-line Figs 1 and 2 .

\section{DISCUSSION}

In this study, we described an intraprocedural FPCTRA and IF technique and demonstrated its usefulness during the TVE. The transvenous approach may have technical difficulties for various reasons. In cases with an occluded accessing route due to thrombosis or hypoplasia, TVE can be more challenging. Breaching the occluded sinus by blinded navigation can be attempted. However, breaching the occluded route is not always feasible, and serious complications, including vessel perforation, can occur. ${ }^{7}$ In addition, delivery of the microcatheter in the wrong direction can occur because breaching the occluded route is always performed by blinded navigation. Moreover, the complexity of head and neck veins that have numerous tributaries and confluences may prevent the microguidewire and microcatheter from advancing in the right direction to the shunt pouch. ${ }^{4}$ Especially, a DAVF involving the ACC can be challenging for navigation of the microcatheter because of complex venous anatomy around the hypoglossal canal. ${ }^{5}$ Additionally, roadmap images during TVE may have low image quality and efficacy due to an overlap of vessels on $2 \mathrm{D}$-DSA. Therefore, blinded microcatheter navigation may be needed, and the microcatheter position for coil insertion can be inappropriately placed. These might lead to unexpected complications or incomplete occlusion. In this study, we used the FPCTRA and IF technique to overcome these obstacles. MPR or a partial MIP image was mainly used for identification of the microcatheter tip with bony landmarks during the blinded navigation. To confirm appropriate microcatheter placement, we used mainly $3 \mathrm{D}$ volume-rendering or MPR fusion images.

This study has some limitations. The retrospective nature and small sample size may not be enough to draw definite conclusions. Moreover, our technique was mainly used in DAVFs involving an ACC or with sinus occlusion needing blinded 
navigation. Other typical lesions could be successfully treated using conventional techniques. Therefore, we do not advocate our technique as a first-line use. Finally, our technique may require more radiation exposure. However, minimizing additional radiation exposure can be accomplished using low-dose protocols. Further large-volume data should be evaluated to confirm the efficacy and safety of our technique.

Intraprocedural use of FPCTRA and IF for TVE might be an effective and safe method to navigate the microcatheter into the targeted shunt pouch, especially in cases that require blinded microcatheter navigation.

\section{REFERENCES}

1. Tanoue $\mathrm{S}$, Kiyosue $\mathrm{H}$, Mori $\mathrm{H}$, et al. Fusion imaging using subtracted and unsubtracted rotational angiography for pretherapeutic evaluation of dural arteriovenous fistulas. Jpn J Radiol 2014;32:600-07 CrossRef Medline

2. Li H, Wan F, Li J, et al. Flat detector computed tomography-based "dual vessel fusion" technique for diagnosis and surgical planning in the management of dural arteriovenous fistula. World Neurosurg 2015;84:520-27 CrossRef Medline
3. Fukuda K, Higashi T, Okawa M, et al. Fusion technique using threedimensional digital subtraction angiography in the evaluation of complex cerebral and spinal vascular malformations. World Neurosurg 2016;85:353-58 CrossRef Medline

4. Choi JH, Shin YS, Kim BS. Making microguidewire loop facilitates navigation through tortuous or abruptly angulated head and neck veins to access cavernous sinus dural arteriovenous fistulas. World Neurosurg 2019;129:e561-65 CrossRef Medline

5. Spittau B, Millán DS, El-Sherifi S, et al. Dural arteriovenous fistulas of the hypoglossal canal: systematic review on imaging anatomy, clinical findings, and endovascular management. J Neurosurg 2015;122:883-903 CrossRef Medline

6. Biondi A, Milea D, Cognard C, et al. Cavernous sinus dural fistulae treated by transvenous approach through the facial vein: report of seven cases and review of the literature. AJNR Am J Neuroradiol 2003;24:1240-46 Medline

7. Cho YD, Rhim JK, Yoo DH, et al. Transvenous microguidewire looping technique for breach of ipsilateral inferior petrosal sinus occlusions en route to cavernous sinus dural arteriovenous fistulas. Interv Neuroradiol 2016;22:590-95 CrossRef Medline

8. Yuki I, Ishibashi T, Dahmani C, et al. Combination of high-resolution cone beam computed tomography and metal artefact reduction software: a new image fusion technique for evaluating intracranial stent apposition after aneurysm treatment. BMJ Case Rep 2019;12: e230687 CrossRef Medline 